\title{
Clinical Use of Continuous Glucose Monitoring in Pediatrics
}

\author{
Rayhan A. Lal, MD ${ }^{1,2}$ and David M. Maahs, MD, PhD ${ }^{1}$
}

Keywords: Continuous glucose monitoring, Hemoglobin A1c, Hyperglycemia, Hypoglycemia, Pediatrics.

\section{Introduction}

T HE FIRST REPORTED clinical use of a continuous glucose monitoring (CGM) system in children was by CossBuet al. in 1999. ${ }^{1}$ They investigated a bedside VIA 1-01G Blood Chemistry monitor for measuring glucose in the pediatric intensive care unit (PICU). The monitor was programmed to draw venous blood, analyze, and record blood glucose samples automatically at predetermined intervals. This system was large, difficult to move, required intravenous access, and took 20 min to set up. Nonetheless, the data provided were comparable to measures by a laboratory glucose analyzer and bedside glucometer and could be used to inform clinical decision-making.

Many advances have occurred since that time to benefit pediatric patients with diabetes. Most recently, the FDA has approved the Dexcom G5 CGM system for use in insulin dosing decisions (nonadjunctive use), ${ }^{2}$ the MiniMed $670 \mathrm{G}$ hybrid closed-loop system which alters insulin delivery based on CGM data for children $\geq 14$ years of age, ${ }^{3}$ and the Abbott FreeStyle Libre Pro for use by healthcare providers in tracking glucose data for those 18 years and over. ${ }^{4}$

In this chapter, published Pediatric data on CGM use will be reviewed in the following areas: type 1 and 2 diabetes mellitus, prediabetes, cystic fibrosis, neonatology, adrenal insufficiency, glycogen storage disease, and critical illness. Data were obtained through review of published works from a PubMed search of "pediatric continuous glucose monitor." We also review pediatric CGM data from artificial pancreas studies, a rapidly evolving field with great promise to reduce burden and improve glucose control in pediatric patients.

\section{Type 1 Diabetes}

The latest position statements by the American Diabetes Association and the International Society of Pediatric and Adolescent Diabetes recommend a Hemoglobin A1c (HbA1c) goal $<7.5 \%$ across all pediatric age groups. ${ }^{5,6}$ This goal challenges even the most motivated, knowledgeable, and socioeconomically privileged patient.

Children with diabetes, in particular, face unique challenges. For example, total daily doses of insulin are proportional to weight, so in children small dose changes can have tremendous impact on glycemic control. Moreover, younger children are by definition hypoglycemic unaware as they are unable to communicate or self-treat low blood sugars. They are completely dependent on different caretakers for disease management. Therefore, CGM has a unique role and distinct challenges and opportunities in the Pediatric population. Modern CGM provides patients and families with accurate and timely glycemic data that may assist in meeting the daily challenges of diabetes and the HbA1c goals measured quarterly.

In 2014 the T1D Exchange conducted a survey on CGM use in the last 30 days. They found CGM was used by $6 \%$ of children $<13$ years old $(n=5027), 4 \%$ of adolescents aged $13-18(n=4855)$, and $6 \%$ of young adults aged $18-25(n=$ 2769). Use was associated with higher education, higher household income, private insurance, longer duration of diabetes, pump use, and lower HbA1c in children. ${ }^{7}$ While it may seem intuitive that providing more glucose data should improve glucose control, a causative relationship has been difficult to establish particularly in children. It should also be noted that this 2014 article and other historic data summarized in this study report use of older generation CGM systems that have been significantly improved in current versions as far as accuracy, comfort, and usability.

Kaufman et al. ${ }^{8}$ performed the first study addressing whether CGM influenced glycemic control in 2001. CGM data from 47 pediatric patients at Children's Hospital of Los Angeles were used by physicians to make insulin dose adjustments. The group found a statistically significant, although clinically small difference in $\mathrm{HbA} 1 \mathrm{c}$ from $8.6 \% \pm 1.5 \%$ at

\footnotetext{
${ }^{1}$ Division of Endocrinology, Department of Pediatrics, Stanford University School of Medicine, Stanford, California

${ }^{2}$ Division of Endocrinology, Department of Medicine, Stanford University School of Medicine, Stanford, California.
} 
baseline to $8.4 \% \pm 1.3 \% .^{8}$ In 2003 Ludvigsson and Hanas performed a controlled crossover study with 27 patients with diabetes age 5-19 in which an open and masked study arm wore CGM for 3 days every 2 weeks. The open study arm received insulin dose adjustment based on the glycemic profiles obtained from the CGM. There was a statistically significant $\mathrm{HbA} 1 \mathrm{c}$ reduction in the open arm from $7.7 \%$ to $7.3 \%$, which was not observed in the masked arm. ${ }^{9}$ A singleblind randomized controlled trial of participants 7-17 years old with CGM also demonstrated a statistically significant decrease in $\mathrm{HbA} 1 \mathrm{c}$ for the intervention group from $8.4 \% \pm$ $0.98 \%$ to $7.8 \% \pm 0.88 \%$. Of note, the difference in HbA1c between the intervention and control groups did not reach statistical significance $(0.61 \% \pm 0.68 \%$ in the intervention group of $n=18$ vs. $0.28 \% \pm 0.78 \%$ in control group of $n=9$; $P=0.18){ }^{10}$

While these initial studies were conducted with early Medtronic CGMs, similar results were reported with a pilot study of the FreeStyle Navigator in 27 children and adolescents (age 417 years) on multiple daily injections. Mean HbA1c level fell from $7.9 \% \pm 1.0 \%$ at baseline to $7.3 \% \pm 0.9 \%$ at 13 weeks. ${ }^{11}$ Meta-analysis in 2012 of seven randomized controlled trials with these previous generation CGM systems did provide evidence of $\mathrm{HbA} 1 \mathrm{c}$ reduction, but the studies included adults and it is difficult to know if the conclusions generalize to the pediatric population or to current CGM systems. ${ }^{12}$

Unfortunately, not all historic studies support the assertion that CGM use improves HbAlc in pediatric age patients. Interpretation of these data requires an understanding of how CGM technology has evolved in the past decade to be more accurate and user friendly. Deiss et al. ${ }^{13}$ performed a doubleblind crossover study in 30 children with diabetes age 2-16 years in 2006. Subjects were randomized to open or masked study arms and had CGM placed for 3 days at the beginning of the study, 3 months, and 6 months. The open group received insulin dose changes based on the CGM data, while the masked group did not. There was no significant change in HbA1c within each group with this limited sensor use. ${ }^{13}$

The JDRF randomized clinical trial from 2008 also failed to show a significant difference in HbA1c between CGM and control for subjects 8-24 years old. ${ }^{14}$ A 2009 secondary analysis of the JDRF cohort revealed that age was strongly positively associated with $\geq 6$ days/week CGM use. In all three age groups (8-14, 15-24, and $\geq 25$ years old), near-daily use of CGM was associated with similar improvements in HbAlc. The authors concluded that additional work is needed to overcome barriers to daily CGM use in children and adolescents. ${ }^{15}$

Another 2010 study showed that the frequency of CGM use among those aged 8-17 years decreases over time, but those using CGM for more than 6 days per week had significant decrease in $\mathrm{HbA} 1 \mathrm{c}$ and greater satisfaction with CGM. ${ }^{16}$ A randomized controlled trial of CGM in those aged 4-9 years for 26 weeks showed a high degree of parental satisfaction, an important metric in this patient population, but no significant change in HbA1c or incidence of severe hypoglycemia. In this group heavy use of CGM ( $>6$ days per week) did not correlate with improved HbA1c. The authors suggested this may have been due to parental fear of hypoglycemia impeding more aggressive insulin changes. ${ }^{17}$ Among individuals $<4$ years there was no difference in $\mathrm{HbA1c}$ after 6 months of use; there was, however, a high degree of parental satisfaction. ${ }^{18}$
Another purported benefit of CGM is avoidance of nocturnal hypoglycemia and increased time in target blood sugar range. Indeed, among the first observations of home CGM use in 2001 was asymptomatic nocturnal hypoglycemia. ${ }^{19}$ Another study conducted by Kaufman revealed that among 47 youths with type 1 diabetes wearing CGM for 167 nights, $27 \%$ had blood glucose $\leq 40 \mathrm{mg} / \mathrm{dL}$ and $35 \% \leq 50 \mathrm{mg} / \mathrm{dL}$. Most of these episodes occurred between 9 PM and $1 \mathrm{AM}^{20}$ In 2003 Amin et al. evaluated 28 prepubertal children $(<12$ years old) on injection regimens who wore sensors for 3 consecutive days and nights and found hypoglycemic prevalence of $10.1 \%$ (mean $2.6 \mathrm{~h} / \mathrm{subject} /$ day) particularly between 4 and 7:30 AM. Risk factors included younger age, greater daily insulin dose, and increased weight. ${ }^{21}$ In a JDRF 2010 masked CGM study $33 \%$ of subjects age $8-14(n=64)$ and $49 \%$ of subjects age 15-24 $(n=42)$ had one or more episodes of nocturnal hypoglycemia in a week. ${ }^{22}$ Ly et al. investigated hypoglycemic unawareness among adolescents with diabetes and found that CGM with a low alarm set at $108 \mathrm{mg} / \mathrm{dL} \mathrm{im-}$ proved counter regulatory hormone response under hypoglycemic clamp compared to standard glucose monitoring. ${ }^{23}$ Data strictly pertaining to CGM intervention in pediatric age patients are more limited and often amalgamated with older populations. A trial in 2011 demonstrated that among 120 patients ages 10-65 years old with well-controlled type 1 diabetes $(\mathrm{HbA} 1 \mathrm{c}<7.5 \%)$, there was a statistically significant decrease in hypoglycemia and HbA1c among CGM users. ${ }^{24}$

With respect to postprandial hyperglycemia, a 2007 study suggested that excursion and rate of glycemic change following breakfast were greater than with lunch or dinner. They also reported no significant difference in postprandial hyperglycemia with multiple daily injections versus pump. ${ }^{25}$ Multiple professional organizations have attempted to provide recommendations based on these numerous studies with data spanning well over a decade (Table 1). ${ }^{26-28}$

One goal of accurate CGM is to inform control systems that modulate insulin delivery using a mechanical pump. The 2010 Sensor Augmented Pump Therapy for A1c Reduction (STAR3) study involved a 1-year, multicenter randomized controlled trial among 156 children (age 7-18 years) and 329 adults with type 1 diabetes and suboptimal control (HbA1c $7.4 \%-9.5 \%$ ). The study compared multiple daily injections and glucometer use with Medtronic insulin pump and CGM. Among children, there was an absolute reduction in $\mathrm{HbA} 1 \mathrm{c}$ of $0.4 \% \pm 0.9 \%$ in the pump-therapy group and an increase of $0.2 \% \pm 1.0 \%$ in the injection-therapy group, for a betweengroup difference favoring the pump-therapy group by $-0.5 \%$ (95\% confidence interval: -0.8 to $-0.2 ; P<0.001){ }^{29}$ The system simply provided sensor data that could be viewed on a pump, insulin delivery was fully controlled by the user.

Since that time effort has been focused on sensor augmented pump or closed-loop systems that take advantage of modern, more accurate, and user-friendly CGM. CGM systems from Abbott, Dexcom, and Medtronic have been investigated in conjunction with a variety of pumps and internal or external control systems. On September 28, 2016 the FDA approved the Medtronic 670G pump and Guardian Sensor 3 for those aged 14 and above with type 1 diabetes. ${ }^{30}$ This represents the first hybrid closed-loop insulin delivery device on the market. In 2017, Garg et al. published an in-home study of the 670G system, including 30 participants of age 14-21 years. They report that HbA1c levels decreased from 
Table 1. Professional Society Guidelines on Continuous Glucose Monitoring in Pediatrics

\begin{tabular}{|c|c|}
\hline Organization & Pediatric recommendations for CGM \\
\hline $\begin{array}{l}\text { American Associate of Clinical } \\
\text { Endocrinologists (AACE) } \\
\text { and American College of } \\
\text { Endocrinology } 26\end{array}$ & $\begin{array}{l}\text { - CGM recommended particularly for patients with history of severe hypoglycemia, } \\
\text { hypoglycemia unawareness, and to assist in correction of hyperglycemia in patients } \\
\text { not at goal. } \\
\text { - CGM users must know basics of sensor insertion, calibration, and real-time data } \\
\text { interpretation. } \\
\text { - Both prevalence and persistent use of CGM are lower in children than adults. More } \\
\text { in-depth training, as well as more frequent follow-up, is recommended to enable } \\
\text { children to adopt the technology more successfully. }\end{array}$ \\
\hline $\begin{array}{l}\text { American Diabetes Association } \\
(\text { ADA })^{27}\end{array}$ & $\begin{array}{l}\text { - Although the evidence for HbA1C lowering is less strong in children, teens, and } \\
\text { younger adults, CGM may be helpful in these groups. Success correlates with } \\
\text { adherence to ongoing use of the device. } \\
\text { - CGM may be a supplemental tool in those with hypoglycemia unawareness or } \\
\text { frequent hypoglycemia. } \\
\text { - Given variable adherence to CGM, assess individual readiness for continuing CGM } \\
\text { use before prescribing. } \\
\text { - When prescribing CGM, robust diabetes education, training, and support are } \\
\text { required for optimal CGM implementation and ongoing use. }\end{array}$ \\
\hline Endocrine Society ${ }^{28}$ & $\begin{array}{l}\text { - Recommend CGM in those with type } 1 \text { diabetes and HbA1c }<7.0 \% \text { because it will } \\
\text { assist in maintaining target HbA1c levels while limiting the risk of hypoglycemia. } \\
\text { - Recommend CGM in those with HbA1c } \geq 7.0 \% \text { who are able to use these devices on } \\
\text { a nearly daily basis. } \\
\text { - No recommendations for or against CGM in children with type } 1 \text { diabetes }<8 \text { years old. } \\
\text { - Recommend treatment guidelines for patients to allow them to take advantage of } \\
\text { CGM data. Suggest intermittent use of CGM for short-term retrospective analysis in } \\
\text { pediatric patients with diabetes and nocturnal hypoglycemia, dawn phenomenon, } \\
\text { postprandial hyperglycemia, hypoglycemic unawareness, and important changes to } \\
\text { their regimen. }\end{array}$ \\
\hline
\end{tabular}

CGM, continuous glucose monitoring; HbA1c, hemoglobin A1c.

$7.7 \% \pm 0.8 \%$ to $7.1 \% \pm 0.6 \%$ and in-target CGM values increased from $60.4 \% \pm 10.9 \%$ to $67.2 \% \pm 8.2 \%{ }^{31}$ We fully expect that these closed-loop innovations will make CGM an integral component of diabetes care.

\section{Type 2 Diabetes}

The first reported use of CGM in pediatric patients with type 2 diabetes was by Boland and Tamborlane in $2000 .^{32}$ They reported on two patients with insulin resistance and demonstrated significant postprandial excursions, but also stability of overnight glucose due to endogenous insulin production. The additional information regarding prandial insulin needs helped the clinician increase meal coverage with insulin. The authors also suggested the use of CGM as an educational and motivational tool to encourage lifestyle modification.

A 2008 study demonstrated frequent hyperglycemia during the day, which correlates with HbA1c, and hypoglycemia during the night for seventeen adolescents with poorly controlled type 2 diabetes. ${ }^{33}$ There is a paucity of data on CGM use in pediatric patients with type 2 diabetes. It remains to be seen whether CGM data can encourage users to make lifestyle modifications to decrease insulin resistance and improve glycemic control.

\section{Prediabetes}

Although no definitive intervention has been established, early identification of hyperglycemia may eventually prove helpful in those at high risk of diabetes, especially as CGM systems become more accurate, less expensive, and burdensome for patient use. In 2014 Steck et al. reported that among
14 asymptomatic children with positive islet autoantibodies, $\geq 18 \%-20 \%$ CGM time spent above $140 \mathrm{mg} / \mathrm{dL}$ predicted progression to diabetes within 6 months. ${ }^{34}$ Helminen et al. compared CGM, HbA1c, and oral glucose tolerance testing (OGTT) for 10 asymptomatic children with genetic predisposition for type 1 diabetes and 2 or more islet autoantibodies against 10 age- and sex-matched controls with genetic predisposition who were antibody negative. Interestingly, no difference was observed in glucose or C-peptide levels during OGTT. However, both HbA1c and CGM values differed significantly between groups. Those with positive autoantibodies had greater mean glucose and greater variation in glucose. The authors concluded that CGM may be a useful early indicator of dysglycemia during prediabetes versus OGTT. ${ }^{35}$

In 2015 Chan et al. collected CGM data on 98 subjects aged $10-18$ years with body mass index $(\mathrm{BMI}) \geq 85$ percentile with A1c $\leq 7.5 \%$. They utilized CGM to help differentiate the predictive power of HbA1c and OGTT for dysglycemia. HbA1c had a greater magnitude of correlation to CGM average glucose, total area under CGM curve (by trapezoidal method), and minimum glucose. OGTT had a greater magnitude of correlation to CGM standard deviation, peak glucose, and time spent in hyperglycemia. They conclude that both HbA1c and OGTT outperform fasting glucose in predicting CGM outcomes, but that they may reflect different underlying pathologic mechanisms for progression to type 2 diabetes. $^{36}$

\section{Cystic Fibrosis}

Cystic Fibrosis-Related Diabetes (CFRD) is a complication that usually develops with age, but can present in 
pediatric age patients. The onset of the disease is insidious, which has prompted annual OGTT screening. In 2008 Franzese et al. compared OGTT and CGM for cystic fibrosis patients 5-20 years old with borderline high glucose during OGTT. CGM and OGTT were in agreement in $43.7 \%$ of the patients. ${ }^{37}$ Schiaffini et al. suggested that CGM excursions $>200 \mathrm{mg} / \mathrm{dL}$ can be a useful tool to predict future impaired glucose tolerance and CFRD. An insidious clinical decline can occur even during the prediabetic state in people with cystic fibrosis, so early detection and treatment can improve long-term health outcomes. ${ }^{38}$

\section{Neonatal Use}

Blood loss from diagnostic sampling is the most frequent cause of anemia in hospitalized infants. Neonates in the neonatal intensive care unit (NICU) frequently have risk factors for hypoglycemia, prompting frequent capillary and venous glucose checks. As early as 2001 Baumeister et al. reported the use of long-term subcutaneous glucose monitoring by microdialysis catheter. They report the safe application of microdialysis catheters in neonates born between gestational age of 30 and 45 weeks for 4-16 days. The method demonstrated $92.3 \%$ sensitivity and $88.1 \%$ specificity for detection of hypoglycemia. $^{39}$ These are especially impressive performance characteristics given CGM accuracy (particularly for hypoglycemia) at the time and results should be confirmed with modern CGM systems.

Although the physiologic significance is unknown, Harris et al. identified many more episodes of low blood glucose using a glucose oxidase sensor than with traditional interstitial glucose monitoring. ${ }^{40}$ In 2013 Beardsall et al. performed a multicenter randomized controlled trial that validated the use of Medtronic CGM from 188 very low birth weight premature infants $(<1000 \mathrm{~g})$. The CGM data correlated well with point of care devices, with minimal bias and accuracy that did not deteriorate over a 7-day period. ${ }^{41}$

Prompted by the safety and validation data in neonates, a group in Japan utilized CGM to detect severe postprandial hypoglycemia associated with dumping syndrome in infants undergoing Nissen fundoplication. They suggest that postsurgical dumping syndrome may be underdiagnosed in infants and that CGM may provide the most sensitive diagnostic tool. ${ }^{42}$

\section{Adrenal Insufficiency}

In 2012 Meyer et al. screened 13 patients with primary adrenal insufficiency due to Addison's disease for hypoglycemia through use of CGM for 3-5 days. They detected a single subject with overnight blood sugar of $46 \mathrm{mg} / \mathrm{dL}$, which prompted an increase in hydrocortisone dosing. The authors conclude that CGM can be used to help detect occult nocturnal hypoglycemia and prevent impaired quality of life and possibly serious adverse events. ${ }^{43}$

Another study by Cambiaso et al. in 2013 evaluated 11 pediatric patients (age 1-16 years) with adrenocorticotropic hormone and growth hormone $(\mathrm{GH})$ deficiency. Subjects utilized CGM for $36 \mathrm{~h}$ and were evaluated for hypoglycemia. There was no relationship between growth hormone dose in GH deficient subjects with and without hypoglycemia (defined as two consecutive glucose values $<50 \mathrm{mg} / \mathrm{dL}$ ). Conversely, there was a statistically significant difference in hydrocortisone dose in patients with secondary adrenal in- sufficiency who experienced hypoglycemia $\left(5.9 \mathrm{mg} / \mathrm{m}^{2} /\right.$ day $)$ and those who did not $\left(8.5 \mathrm{mg} / \mathrm{m}^{2} /\right.$ day $)$. As in the first study the authors infer that CGM can be used to detect asymptomatic nocturnal hypoglycemia, which may prompt physicians to increase hydrocortisone dosing. ${ }^{44}$

\section{Glycogen Storage Disorders}

Glycogen storage disease type I (GSD I) is an autosomal recessive metabolic disease effecting the enzyme glucose-6phosphatase. Clinically, it is characterized by fasting hypoglycemia within 3-4 h after a meal. Kasapkara et al. placed 16 children with GSD I on CGM for $72 \mathrm{~h}$ and then used the data to inform dietary changes. Following the intervention there was a significant reduction in CGM recorded hypoglycemia and liver size and improvement in lactic acidemia and hyperlipidemia. The authors conclude that even this timelimited intervention can have meaningful impact for longterm management of patients with GSD I. ${ }^{45}$

\section{Critical IIIness}

In the PICU there are many fluctuating physiologic parameters which can adversely affect the performance of glucose oxidase based sensors. In addition, awareness of acetaminophen interference with these systems is particularly important in an ICU setting. ${ }^{46,47}$ Branco et al. explored physiologic aberrations in the use of CGM in the PICU. Specifically, they examined the correlation between glucose measured from arterial blood, point of care glucometer, and CGM in 14 children ages 1 month to 16 years requiring mechanical ventilation with at least two organ system failures. Measures of mean absolute relative difference (MARD) appeared to correlate with significant acidosis and therapeutic hypothermia, which the authors felt may limit the use of CGM. ${ }^{48}$ Using statistical definitions (Bland-Altman and Clarke error grid analysis) both this study and another demonstrated clinically acceptable correlation despite the aforementioned limitations. ${ }^{49}$ There is sufficient trust in the technology that the 2017 HALF-PINT study investigating tight glycemic control in critically ill children used CGM to signal impending hypoglycemia. ${ }^{50}$

\section{Special Considerations in Pediatrics}

A multicenter observational study performed on 149 children and adolescents with type 1 diabetes in 2015 revealed that the majority of subjects discontinued CGM use, even when financial considerations were not an issue. Only $38 \%$ used CGM regularly more than $75 \%$ of the time, despite the finding that those using CGM consistently had better glycemic control during the year compared to intermittent users. Consistent use correlated with younger age and more frequent finger-stick glucose checks at baseline. The authors conclude that providers should be aware that CGM is most useful in compliant patients. ${ }^{51}$

Naranjo et al. reported in 2016 that those using pumps, CGMs, or sensor augmented pumps had more positive attitudes about diabetes technology than those on multiple daily injections and conventional glucose monitoring. ${ }^{52}$ Among all participants in the JDRF CGM study group common barriers to continued use include insertion pain, system alarms, and body issues related to the need to wear the device, ${ }^{53}$ although this was with early generation systems. 
Certain concerns are more specific to the pediatric age patient. Children with diabetes spend $4-7 \mathrm{~h}$ in school per day during which time they have decreased diabetes supervision. Based on one survey, $75 \%$ of students, $70 \%$ of parents, and $51 \%$ of teachers found CGM useful at school. More students felt the devices to be disruptive than parents or teachers. The authors suggest educational materials be provided to teachers to increase their comfort. ${ }^{54}$ With the recent FDA approval of insulin dosing based on Dexcom CGM and the Medtronic $670 \mathrm{G}$ system, advocacy and education for use of these systems in school will be required.

Pediatric use of CGM can be limited by body surface area in smaller children, ambient temperature/humidity, and physical activity. Sensor adhesion appears significant for improving long-term adherence. Englert et al. suggest adhesive wipes, liquid adhesive, transparent dressings, tape, and wraps to combat the physical factors that impair good CGM placement. ${ }^{55}$ During hybrid closed-loop studies it was felt that calibrating CGM to interstitial glucose obtained from a second drop of blood (rather than the first) reduced errors with MARD of $10.8 \%$ versus laboratory standard and $12.6 \%$ versus glucometer. ${ }^{56}$

CGMs generate a tremendous, at times overwhelming, quantity of data. The information must be processed and analyzed to have meaningful impact. Parents report high levels of satisfaction when they have the ability to monitor their children's blood sugars remotely. ${ }^{57}$ Frequently, this provides reassurance regarding hypoglycemia. In addition, the rate of glucose change, in the form of arrows, can allow one to act reflexively in anticipation of high or low blood sugars. While these techniques do assist in the short term, assessing prior data to make long-term insulin dose changes can obviate the need for these reflexive behaviors by increasing time in target range. Automated insulin dosing systems that integrate CGM data will also address this need.

There are many ways of retrospectively visualizing glucose data, for example, the ambulatory glucose profile (AGP). A recent analysis by Forlenza revealed differences in the average AGP glycemic patterns for the JDRF-CGM dataset when stratified by age group (8-14, 15-24, and $\geq 25$ years) and $\mathrm{HbA1c}$. They report that for a given HbA1c level, all age groups were significantly different, with older patients having lower averages and less variability than younger patients. ${ }^{58}$

We encourage patients and families to actively download CGM statistics, evaluate the data, and send it to their diabetes care team. Passively moving CGM data to physicians for analysis is another active area of research. Dexcom's CGM can send glycemic data to an iOS app through Bluetooth which can then be exported to the electronic medical record system without patient intervention. ${ }^{59}$ As data accumulate we can now analyze trends on large patient populations.

\section{Relative Performance}

In 2014 Damiano et al. published a head-to-head comparison of the Abbott FreeStyle Navigator (Navigator), Dexcom G4 Platinum (G4P), and Medtronic Enlite (Enlite) CGM in participants over the age of $12 .{ }^{60}$ Previously, the G4P received a software update which has been shown to improve MARD from $17 \%$ to $10 \%$ over a 7 -day period in children aged 2-17 years. ${ }^{61}$ The G4P had an aggregate MARD of $10.8 \% \pm 9.9 \%$, which was not significantly different from the Navigator at $12.3 \% \pm 12.1 \%$. The Enlite had significantly less accurate performance with an aggregate MARD of $17.9 \% \pm 15.8 \%$. The average MARD for experiments in adolescent subjects was lower than in adult subjects for the $\mathrm{Na}$ vigator and G4P, while there was no difference for Enlite.

Sensor technology and accuracy continue to improve with time. CGM accuracy is a requirement for closed-loop insulin delivery. Prepublication online data from Medtronic for children and adults suggest that the Guardian Sensor 3 has a MARD of $9.64 \%$ when calibrating 3-4 times/day and $10.55 \%$ when calibrating 1-2 times/day. ${ }^{62}$ Abbott's FreeStyle Libre system, recently approved in the United States for professional use, has a reported MARD of $11.4 \%$ (all ages) versus glucometer and does not require finger-stick calibration. ${ }^{63}$ Rather than providing continuous glucose reading to a receiver, the Libre system provides on-demand glucose data. Another innovation of the Libre system is that no user calibration is required, removing an often cited burden of CGM use by patients and families. Abbott and Dexcom devices are approved to dose insulin based on CGM readings, a tremendous step forward which illustrates the significant improvements in CGM accuracy.

\section{Future Directions}

As described CGM has already been explored in a variety of pediatric clinical settings. The technology improves parental satisfaction, reduces hypoglycemia, and has a positive impact on overall glycemic control when used consistently. The newest iterations of CGM products have improved interfaces and are now accurate enough to be used in closedloop insulin delivery systems. We expect that as technology improves, CGM will become less invasive, less burdensome, and more beneficial. Alternately, long-term implantable CGMs may also have a role in pediatrics in the future, although more research is required. ${ }^{64,65}$ As the burden of wearing CGM decreases and accuracy increases, we anticipate CGM becoming an indispensable tool that succeeds the finger stick in a multitude of pediatric clinical settings.

\section{Author Disclosure Statement}

No competing financial interests exist.

\section{References}

1. Coss-Bu JA, Jefferson LS, Stone-McCord S, et al.: Evaluation of a real-time blood glucose monitor in children with diabetic ketoacidosis. Diabetes Res Clin Pract 1999; 44:175-181.

2. Thibault M: Dexcom's Bid for Non-Adjunctive Label Blessed by Panel. MDDI. N.p., July 22, 2016. http:// www.mddionline.com/article/dexcoms-bid-non-adjunctivelabel-blessed-07-22-16 (accessed February 20, 2017).

3. Tilleskjor S: Breaking News: FDA Approves the MiniMed 670G System, World's First Hybrid Closed Loop System. Between The Lines Blog | Medtronic Diabetes. Medtronic. October 15, 2016. https://www.medtronicdiabetes.com/ blog/introducing-the-minimed-670g-system/ (accessed February 20,2017$)$.

4. Schmidt M: Freestyle Libre Pro Receives FDA Approval. Diabetes 365. Diabetes 365 Publisher. October 10, 2016. https://www.diabetes365.org/freestyle-libre-pro-receivesfda-approval/ (accessed February 20, 2017). 
5. Chiang JL, Kirkman MS, Laffel LM, Peters AL: Type 1 diabetes through the life span: a position statement of the American Diabetes Association. Diabetes Care 2014;37: 2034-2054.

6. Rewers MJ, Pillay K, de Beaufort C, et al.: International Society for Pediatric and Adolescent Diabetes. ISPAD Clinical Practice Consensus Guidelines 2014. Assessment and monitoring of glycemic control in children and adolescents with diabetes. Pediatr Diabetes 2014;15 Suppl 20: 102-114.

7. Wong JC, Foster NC, Maahs DM, et al.: T1D Exchange Clinic Network. Real-time continuous glucose monitoring among participants in the T1D Exchange clinic registry. Diabetes Care 2014;37:2702-2709.

8. Kaufman FR, Gibson LC, Halvorson M, et al.: A pilot study of the continuous glucose monitoring system: clinical decisions and glycemic control after its use in pediatric type 1 diabetic subjects. Diabetes Care 2001;24:2030-2034.

9. Ludvigsson J, Hanas R: Continuous subcutaneous glucose monitoring improved metabolic control in pediatric patients with type 1 diabetes: a controlled crossover study. Pediatrics 2003;111(5 Pt 1):933-938.

10. Lagarde WH, Barrows FP, Davenport ML, et al.: Continuous subcutaneous glucose monitoring in children with type 1 diabetes mellitus: a single-blind, randomized, controlled trial. Pediatr Diabetes 2006;7:159-164.

11. Weinzimer S, Xing D, Tansey M, et al.: FreeStyle navigator continuous glucose monitoring system use in children with type 1 diabetes using glargine-based multiple daily dose regimens: results of a pilot trial Diabetes Research in Children Network (DirecNet) Study Group. Diabetes Care 2008;31:525-527.

12. Szypowska A, Ramotowska A, Dzygalo K, Golicki D: Beneficial effect of real-time continuous glucose monitoring system on glycemic control in type 1 diabetic patients: systematic review and meta-analysis of randomized trials. Eur J Endocrinol 2012;166:567-574.

13. Deiss D, Hartmann R, Schmidt J, Kordonouri O: Results of a randomised controlled cross-over trial on the effect of continuous subcutaneous glucose monitoring (CGMS) on glycaemic control in children and adolescents with type 1 diabetes. Exp Clin Endocrinol Diabetes 2006;114:63-67.

14. Juvenile Diabetes Research Foundation Continuous Glucose Monitoring Study Group, Tamborlane WV, Beck RW, et al.: Continuous glucose monitoring and intensive treatment of type 1 diabetes. N Engl J Med 2008;359:1464-1476.

15. Juvenile Diabetes Research Foundation Continuous Glucose Monitoring Study Group, Beck RW, Buckingham B, et al.: Factors predictive of use and of benefit from continuous glucose monitoring in type 1 diabetes. Diabetes Care 2009;32:1947-1953.

16. Chase HP, Beck RW, Xing D, et al.: Continuous glucose monitoring in youth with type 1 diabetes: 12-month followup of the Juvenile Diabetes Research Foundation continuous glucose monitoring randomized trial. Diabetes Technol Ther 2010;12:507-515.

17. Mauras N, Beck R, Xing D, et al.: A randomized clinical trial to assess the efficacy and safety of real-time continuous glucose monitoring in the management of type 1 diabetes in young children aged 4 to $<10$ years. Diabetes Care 2012;35:204-210.

18. Tsalikian E, Fox L, Weinzimer S, et al.: Feasibility of prolonged continuous glucose monitoring in toddlers with type 1 diabetes. Pediatr Diabetes 2012;13:301-307.
19. Chase HP, Kim LM, Owen SL, et al.: Continuous subcutaneous glucose monitoring in children with type 1 diabetes. Pediatrics 2001;107:222-226.

20. Kaufman FR, Austin J, Neinstein A, et al.: Nocturnal hypoglycemia detected with the Continuous Glucose Monitoring System in pediatric patients with type 1 diabetes. J Pediatr 2002;141:625-630.

21. Amin R, Ross K, Acerini CL, et al.: Hypoglycemia prevalence in prepubertal children with type 1 diabetes on standard insulin regimen: use of continuous glucose monitoring system. Diabetes Care 2003;26:662-667.

22. Juvenile Diabetes Research Foundation Continuous Glucose Monitoring Study Group: Prolonged nocturnal hypoglycemia is common during 12 months of continuous glucose monitoring in children and adults with type 1 diabetes. Diabetes Care 2010;33:1004-1008.

23. Ly TT, Hewitt J, Davey RJ, et al.: Improving epinephrine responses in hypoglycemia unawareness with real-time continuous glucose monitoring in adolescents with type 1 diabetes. Diabetes Care 2011;34:50-52.

24. Battelino T, Phillip M, Bratina N, et al.: Effect of continuous glucose monitoring on hypoglycemia in type 1 diabetes. Diabetes Care 2011;34:795-800.

25. Gandrud LM, Xing D, Kollman C, et al.: The Medtronic Minimed Gold continuous glucose monitoring system: an effective means to discover hypo- and hyperglycemia in children under 7 years of age. Diabetes Technol Ther 2007; 9:307-316.

26. Bailey TS, Grunberger G, Bode BW, et al.: American Association of Clinical Endocrinologists and American College of Endocrinology 2016 Outpatient Glucose Monitoring Consensus Statement. Endocr Pract 2016;22:231-261.

27. American Diabetes Association: 6. Glycemic targets. Diabetes Care 2017;40 Suppl 1:S48-S56.

28. Klonoff DC, Buckingham B, Christiansen JS, et al.: Continuous glucose monitoring: an Endocrine Society Clinical Practice Guideline. J Clin Endocrinol Metab 2011;96: 2968-2979.

29. Bergenstal RM, Tamborlane WV, Ahmann A, et al.: Effectiveness of sensor-augmented insulin-pump therapy in type 1 diabetes. N Engl J Med 2010;363:311-320.

30. Smalley E: Medtronic automated insulin delivery device gets FDA nod. Nat Biotechnol 2016;34:1220.

31. Garg SK, Weinzimer SA, Tamborlane WV, et al.: Glucose outcomes with the in-home use of a hybrid closed-loop insulin delivery system in adolescents and adults with type 1 diabetes. Diabetes Technol Ther 2017;19:155-163.

32. Boland EA, Tamborlane WV: Continuous glucose monitoring in youth with type 2 diabetes: overcoming barriers to successful treatment. Diabetes Technol Ther 2000;2 Suppl 1:S53-S59.

33. Lu H, Castells S, Hagerty D, Quintos JB: Study of glucose profiles with continuous glucose monitoring in adolescents with poorly controlled type 2 diabetes mellitus. J Pediatr Endocrinol Metab 2008;21:729-736.

34. Steck AK, Dong F, Taki I, et al.: Early hyperglycemia detected by continuous glucose monitoring in children at risk for type 1 diabetes. Diabetes Care 2014;37:2031-2033.

35. Helminen O, Pokka T, Tossavainen P, et al.: Continuous glucose monitoring and $\mathrm{HbA} 1 \mathrm{c}$ in the evaluation of glucose metabolism in children at high risk for type 1 diabetes mellitus. Diabetes Res Clin Pract 2016;120:89-96.

36. Chan CL, Pyle L, Newnes L, et al.: Continuous glucose monitoring and its relationship to hemoglobin A1c and oral 
glucose tolerance testing in obese and prediabetic youth. J Clin Endocrinol Metab 2015;100:902-910.

37. Franzese A, Valerio G, Buono P, et al.: Continuous glucose monitoring system in the screening of early glucose derangements in children and adolescents with cystic fibrosis. J Pediatr Endocrinol Metab 2008;21:109-116.

38. Schiaffini R, Brufani C, Russo B, et al.: Abnormal glucose tolerance in children with cystic fibrosis: the predictive role of continuous glucose monitoring system. Eur J Endocrinol 2010;162:705-710.

39. Baumeister FA, Rolinski B, Busch R, Emmrich P: Glucose monitoring with long-term subcutaneous microdialysis in neonates. Pediatrics 2001;108:1187-1192.

40. Harris DL, Battin MR, Weston PJ, Harding JE: Continuous glucose monitoring in newborn babies at risk of hypoglycemia. J Pediatr 2010;157:198.e1-202.e1.

41. Beardsall K, Vanhaesebrouck S, Ogilvy-Stuart AL, et al.: Validation of the continuous glucose monitoring sensor in preterm infants. Arch Dis Child Fetal Neonatal Ed 2013;98: F136-F140.

42. Ueda K, Mizumoto H, Shibata H, et al.: Continuous glucose monitoring for suspected dumping syndrome in infants after Nissen fundoplication. Pediatr Int 2013;55:782-785.

43. Meyer G, Hackemann A, Reusch J, Badenhoop K: Nocturnal hypoglycemia identified by a continuous glucose monitoring system in patients with primary adrenal insufficiency (Addison's disease). Diabetes Technol Ther 2012;14:386-388.

44. Cambiaso P, Schiaffini R, Pontrelli G, et al.: Nocturnal hypoglycaemia in ACTH and GH deficient children: role of continuous glucose monitoring. Clin Endocrinol (Oxf) 2013; 79:232-237.

45. Kasapkara ÇS, Cinasal Demir G, Hasanoğlu A, Tümer L: Continuous glucose monitoring in children with glycogen storage disease type I. Eur J Clin Nutr 2014;68:101-105.

46. Maahs DM, DeSalvo D, Pyle L, et al.: Effect of acetaminophen on CGM glucose in an outpatient setting. Diabetes Care 2015;38:e158-e159.

47. Basu A, Veettil S, Dyer R, et al.: Direct evidence of acetaminophen interference with subcutaneous glucose sensing in humans: a pilot study. Diabetes Technol Ther 2016;18 Suppl 2:S243-S247.

48. Branco RG, Chavan A, Tasker RC: Pilot evaluation of continuous subcutaneous glucose monitoring in children with multiple organ dysfunction syndrome. Pediatr Crit Care Med 2010;11:415-419.

49. Bridges BC, Preissig CM, Maher KO, Rigby MR: Continuous glucose monitors prove highly accurate in critically ill children. Crit Care 2010;14:R176.

50. Agus MS, Wypij D, Hirshberg EL, et al.: Tight glycemic control in critically ill children. N Engl J Med 2017;376: 729-741.

51. Rachmiel M, Landau Z, Boaz M, et al.: The use of continuous glucose monitoring systems in a pediatric population with type 1 diabetes mellitus in real-life settings: the AWeSoMe Study Group experience. Acta Diabetol 2015;52: 323-329.

52. Naranjo D, Tanenbaum ML, Iturralde E, Hood KK: Diabetes technology: uptake, outcomes, barriers, and the intersection with distress. J Diabetes Sci Technol 2016;10: 852-858.
53. Tansey M, Laffel L, Cheng J, et al.: Satisfaction with continuous glucose monitoring in adults and youths with type 1 diabetes. Diabet Med 2011;28:1118-1122.

54. Benassi K, Drobny J, Aye T: Real-time continuous glucose monitoring systems in the classroom/school environment. Diabetes Technol Ther 2013;15:409-412.

55. Englert K, Ruedy K, Coffey J, et al.: Skin and adhesive issues with continuous glucose monitors: a sticky situation. J Diabetes Sci Technol 2014;8:745-751.

56. Ly TT, Roy A, Grosman B, et al.: Day and night closedloop control using the integrated medtronic hybrid closedloop system in type 1 diabetes at diabetes camp. Diabetes Care 2015;38:1205-1211.

57. Oron T, Farfel A, Muller I, et al.: A remote monitoring system for artificial pancreas support is safe, reliable, and user friendly. Diabetes Technol Ther 2014;16:699-705.

58. Forlenza GP, Pyle LL, Maahs DM, Dunn TC: Ambulatory glucose profile analysis of the juvenile diabetes research foundation continuous glucose monitoring datasetApplications to the pediatric diabetes population. Pediatr Diabetes 2016. November 23, doi: 10.1111/pedi.12474.

59. Kumar RB, Goren ND, Stark DE, et al.: Automated integration of continuous glucose monitor data in the electronic health record using consumer technology. J Am Med Inform Assoc 2016;23:532-537.

60. Damiano ER, McKeon K, El-Khatib FH, et al.: A comparative effectiveness analysis of three continuous glucose monitors: the Navigator, G4 Platinum, and Enlite. J Diabetes Sci Technol 2014;8:699-708.

61. Laffel L: Improved accuracy of continuous glucose monitoring systems in pediatric patients with diabetes mellitus: results from two studies. Diabetes Technol Ther 2016;18 Suppl 2:S223-S233.

62. Diabetes Daily Staff: Medtronic's New 670G: Answers to Your Frequently Asked Questions. Diabetes Daily. October 11, 2016. https://www.diabetesdaily.com/blog/medtronics-new-670ganswers-to-your-frequently-asked-questions-314306/ (accessed February 20, 2017).

63. Data on File, Abbott Diabetes Care, Inc. Clinical Report: Evaluation of the Accuracy of the Abbott Sensor-Based Interstitial Glucose Monitoring System 2014. 2014. From Abbott's website: https://freestylediabetes.co.uk/freestylethinking/post/accuracy (accessed February 20, 2017).

64. Gough DA, Kumosa LS, Routh TL, et al.: Function of an implanted tissue glucose sensor for more than 1 year in animals. Sci Transl Med 2010;2:42ra53.

65. Kropff J, Choudhary P, Neupane S, et al.: Accuracy and longevity of an implantable continuous glucose sensor in the PRECISE study: a 180-day, prospective, multicenter, pivotal trial. Diabetes Care 2017;40:63-68.

Address correspondence to: Rayhan A. Lal, MD

Division of Endocrinology Department of Pediatrics Stanford University School of Medicine Room G-313 Medical Center 300 Pasteur Drive Stanford, CA 94305

E-mail: inforay@stanford.edu 